\title{
Caries e índice de higiene oral en niños con diabetes mellitus tipo 1
}

\author{
XIMENA MIRANDA O..$^{1}$, JORGE TRONCOSO P. ${ }^{2}$, CAROLINA RODRÍGUEZ S. ${ }^{3}$, \\ PEDRO ARAVENA T. ${ }^{3}$, PATRICIA JIMÉNEZ DEL R. ${ }^{4}$
}

1. Cirujano-Dentista. Centro de Salud Familiar "Dr. Enrique Dintrans", Rancagua. Posta de Salud Rural Olivar Bajo.

2. Cirujano-Dentista. Programa Especialidad Implantología Bucomaxilofacial, Universidad de Chile.

3. Escuela de Odontología, Facultad de Medicina, Universidad Austral de Chile.

4. Odontopediatra, Directora Programa Especialidad Odontopediatría. Escuela de Odontología, Universidad de Talca de Chile.

\begin{abstract}
Dental caries and hygiene oral index in children with diabetes mellitus type 1

Introduction: Type 1 Diabetes mellitus (DM1) undeniably affects oral health. High glucose levels may be associated with low salivary flow, accumulation of plaque and tooth decay. The objective of this study is to determine the prevalence of caries and Oral Hygiene Index (OHI) in children with DM1 in Maule Region, Chile during 2008. Patients and Method: A cross-sectional study was designed to examine patients under 15 years old with DM1, who were admitted to Talca Regional Hospital. History of caries (decayed, sealed and lost teeth) was registered according to the COPD-dmft index, OHI ( $>1.2$ high accumulation of plaque) and potential of cariogenic diet (high, medium or low). The statistical relationship between caries history and gender, OHI and diet was analyzed. Results: 25 children cases with DM1 were studied; the prevalence of caries was $92 \%$, which was mostly in boys $(\mathrm{p}=0.03)$ and associated with poor hygiene $(\mathrm{p}<0.01)$. The average COPD- ceod index was $2.96 \pm 2.33$ and $2 \pm 2.64$ respectively. The average OHI was $1.8 \pm 2.64,84 \%$ corresponded to bad oral hygiene. 21 children $(84 \%)$ had a diet with low cariogenic potential, not associated to the presence of caries $(p=0.17)$. Conclusion: The children oral health was precarious and it may be associated directly with poor oral hygiene and not with the food consumed.
\end{abstract}

(Key words: Type 1 Diabetes mellitus, dental caries, COPD index, cariogenic diet, oral hygiene).

Rev Chil Pediatr 2013; 84 (5): 527-531

Recibido el 5 de mayo de 2012, devuelto para corregir el 5 de septiembre de 2012, segunda versión 9 de enero de 2013, tercera versión 13 de julio de 2013, aceptado para publicación el 26 de agosto de 2013.

Los autores aprueban el manuscrito final para su envío y revisión en Revista Chilena de Pediatría, manifestando que no existe conflicto de interés con centros de salud, empresas farmacéuticas, de tipo contractual o éticas. Así mismo, declaran que este estudio se llevó a cabo con financiamiento particular y propio, sin apoyo de alguna compañía o empresa.

Este trabajo cumple con los requisitos sobre consentimiento /asentimiento informado, comité de ética, financiamiento, estudios animales y sobre la ausencia de conflictos de intereses según corresponda.

\section{Correspondencia a:}

Pedro Aravena T.

E-mail: paravena@uach.cl 


\section{RESUMEN}

Introducción: La diabetes mellitus tipo 1 (DM1) influye en la salud oral. Una alta glicemia podría asociarse a una disminución del flujo salival, acúmulo de placa bacteriana y formación de caries. Nuestro objetivo fue determinar la prevalencia de caries e índice de higiene oral (IHO) en niños con DM1 de la Región del Maule-Chile del año 2008. Pacientes y Método: Se diseñó un estudio de corte transversal. Se examinó población menor de 15 años con DM1 del Hospital Regional de Talca. Se registró historia de caries (dientes cariados/obturados/ perdidos) según índice COPD-ceod; IHO (>1,2 alto acúmulo de placa) y potencial cariogénico de dieta (alto/ medio/bajo). Se analizó la relación estadística entre historia de caries con sexo, IHO y dieta. Resultados: 25 niños con DM1 fueron analizados. La prevalencia de caries fue $92 \%$ siendo mayormente en hombres $(p=0,03)$ y asociada a una mala higiene $(\mathrm{p}<0,01)$. El promedio COPD-ceod fue $2,96 \pm 2,33$ y $2 \pm 2,64$ respectivamente. El IHO promedio fue $1,8 \pm 2,64$ siendo $84 \%$ tipo mala. 21 niños $(84 \%)$ tuvieron un potencial cariogénico de dieta bajo, no asociándose a la presencia de caries $(\mathrm{p}=0,17)$. Conclusión: La salud oral de los niños DM1 fue precaria pudiendo corresponder directamente a la mala higiene oral y no con la dieta consumida.

(Palabras clave: Diabetes mellitus tipo 1, caries dental, índice COPD, dieta cariogénica, higiene oral).

Rev Chil Pediatr 2013; 84 (5): 527-531

\section{Introducción}

La Diabetes mellitus tipo 1 (DM1) es una enfermedad autoinmune producida por la destrucción progresiva de las células beta pancreáticas. Corresponde al $70-80 \%$ de todos los casos de DM en niños, observándose una incidencia de 6,58 por 100.000 menores de 15 años ${ }^{1-5}$ y registrándose una marcada tendencia al aumento en la incidencia en niños de 0 a 4 años, llevando a Chile a ser clasificado desde un país de baja incidencia a uno con incidencia intermedia ${ }^{6}$.

La DM1 es una de las enfermedades sistémicas crónicas con repercusiones a nivel de la salud oral. El aumento sostenido de altas concentraciones sanguíneas de glucosa genera disminución del flujo salival, disminución de la respuesta vascular periférica ${ }^{7}$ contribuyendo a la acumulación de placa bacteriana y la formación de tártaro, al desarrollo de caries, halitosis y enfermedad periodontal ${ }^{8}$. Los niños y adolescentes con DM1 suelen presentar en términos generales mayor respuesta de los tejidos periodontales a la placa bacteriana, lo que se expresa en mayor inflamación gingival y una mayor predisposición a padecer enfermedades periodontales más graves ${ }^{5,9,10}$.

Una de las medidas que facilita el control de la enfermedad es el riguroso control de la alimentación basado en el tipo de aporte ca- lórico según la edad, actividad física y el esquema insulínico empleado ${ }^{1,5}$. Esta alimentación debiera ser controlada en el consumo de carbohidratos, pudiendo actuar como un factor protector contra la caries al considerarse como una dieta poco cariogénica ${ }^{11}$.

A la fecha, no existen reportes publicados asociados a base de datos de revistas con comité editorial donde se observe el estado de salud oral de pacientes con DM1. El objetivo de esta investigación fue determinar la prevalencia de historia de caries, el acúmulo de placa bacteriana registrado por el Índice de Higiene Oral (IHO) y el grado de cariogenicidad de la dieta consumida en la población de niños con DM1 de la Región del Maule-Chile, controlados en el Hospital Regional de Talca durante el año 2008.

\section{Pacientes y Método}

Estudio de corte transversal. La población de estudio fueron niños menores de 15 años diagnosticados con DM1 de la Región del Maule derivados y controlados por el servicio de Medicina Interna del Centro de Diagnóstico y Tratamiento del Hospital Regional de Talca durante el año 2008. Se seleccionaron para su examen oral aquellos niños menores a 15 años, que presentaron al momento del estudio índices de glicemia en ayunas igual o mayor a 
$126 \mathrm{mg} / \mathrm{dL}^{1}$; donde los padres autorizaran su participación mediante lectura y aprobación del Consentimiento Informado y el paciente mediante asentimiento cuando correspondiese, a la participación en el estudio. Tanto el diseño de estudio como el Consentimiento Informado fueron aprobados por el Comité de Ética del Servicio de Salud del Maule.

La investigadora principal (XM) registró las siguientes variables: el sexo (hombre/mujer); la edad (en años); presencia de caries (sí/no) considerada como lesión con cavidad evidente y detectable mediante examen visual; historia de caries dental medida a través de los índices COPD y ceod las cuales registran, respectivamente, la prevalencia de caries en dentición permanente ${ }^{12}$ y decidua $^{13}$ en un individuo mediante el número de dientes cariados, obturados y perdidos o con extracción indicada por motivo de caries ${ }^{14}$. Para obtener el índice promedio de caries en nuestro estudio, se sumó el total de la historia de caries de los pacientes dividido por el número total de la muestra; grado de acúmulo de placa bacteriana mediante el Índice de Higiene Oral de Green y Vermillion simplificado y modificado $(<1,2$ bajo acúmulo de placa; $>1,2$ alto acúmulo de placa $)^{15}$ y dieta cariogénica obtenida a través un diario dietético validado por la Universidad de Chile, clasificando el riesgo de cariogenicidad de ésta como riesgo bajo (10-33) moderado (34-79) y alto (80-144), según número de momentos de azúcar consumido diariamente por una semana ${ }^{16}$. Para el control de sesgo, el examinador fue previamente calibrado con la Odontóloga especialista en Odontopediatría del centro de salud examinando 20 niños, obteniendo un índice Kappa de 1 para el índice COPD; 0,92 en el ceod y de 0,92 para índice de Higiene.

El número de pacientes a evaluar se obtuvo mediante cálculo de tamaño muestral, considerando que la Región del Maule cuenta con 240.494 habitantes menores de 15 años ${ }^{17}$; considerando una prevalencia arbitraria a observar del $50 \%$ de niños DM1 con caries y estimando en Chile una tasa de pacientes con DM1 de $0,5 \%{ }^{1}$ con un margen de error del 7\% y un nivel de significancia del $5 \%$, el número estimado a evaluar fue de 37 pacientes.

De los datos obtenidos se analizó mediante estadística descriptiva (promedio \pm desviación estándar) y la asociación entre la presencia de caries y el género de los niños, el tipo de IHO y la dieta cariogénica mediante test chi-cuadrado $(p<0,05)$. La tabulación de los datos se realizó con el software MS Excel 2003 (Microsoft, USA) y el análisis estadístico Stata 10.0.

\section{Resultados}

En el año 2008 se registraron 37 niños menores de 15 años con diagnóstico de DM1, de los cuales solo $25(67,5 \%)$ cumplieron con los criterios de selección; con un promedio de edad de 11,3 años de los cuales 17 (68\%) fueron hombres. La prevalencia de caries fue de $92 \%$ siendo estadísticamente mayor en hombres $(\mathrm{p}=0,03)$. El índice promedio COPD y ceod fue $2,96 \pm 2,33$ y $2 \pm 2,64$, respectivamente. Se obtuvo un IHO promedio 1,8 $\pm 0,84$ siendo $84 \%$ de tipo malo, observándose una asociación estadísticamente significativa con la presencia de caries $(\mathrm{p}<0,01)$. Respecto al potencial cariogénico de dieta, el valor promedio de las encuestas fue de 28,7 \pm 7,9 correspondiendo a un potencial cariogénico bajo. No hubo registro de dieta con potencial cariogénico alto. No se observó una asociación significativa entre el tipo de dieta y la presencia de caries en los niños con DM1 $(p=0,17)$. Los resultados se detallan en tabla 1 .

Tabla 1. Promedios aritméticos y desviación estándar (DE) de índices COPD, ceod, índice de higiene oral y dieta cariogénica de pacientes con diabetes mellitus tipo 1 del Hospital Regional de Talca año $2008(n=25)$

\begin{tabular}{|c|c|c|c|}
\hline Variable & Tipo & $\begin{array}{c}\text { n pacientes } \\
(\%)\end{array}$ & $\begin{array}{l}\text { Promedio } \\
\text { (DE) }\end{array}$ \\
\hline Presencia caries & & $23 \quad(92)$ & - \\
\hline Historia de caries & $\begin{array}{l}\text { COPD } \\
\text { ceod }\end{array}$ & $\begin{array}{l}25(100) \\
13 \quad(52)\end{array}$ & $\begin{array}{lr}2,96 & (2,33) \\
2 & (2,64)\end{array}$ \\
\hline Índice higiene oral & $\begin{array}{l}\text { Buena } \\
\text { Mala }\end{array}$ & $\begin{array}{rr}4 & (16) \\
21 & (84)\end{array}$ & $\begin{array}{r}0,83(0,13) \\
2(0,35)\end{array}$ \\
\hline $\begin{array}{l}\text { Potencial cariogénico } \\
\text { dieta }\end{array}$ & $\begin{array}{l}\text { Bajo } \\
\text { Moderado } \\
\text { Alto }\end{array}$ & $\begin{array}{rr}21 & (84) \\
4 & (16) \\
0 & (0)\end{array}$ & $\begin{array}{l}27,4(3,17) \\
35,5(0,57) \\
0\end{array}$ \\
\hline
\end{tabular}




\section{Discusión}

En pacientes pediátricos diagnosticados con DM1 atendidos en el Hospital Regional de Talca, se observó una alta prevalencia de caries, un mal IHO y bajo potencial cariogénico en su dieta.

Los índices de caries observados en nuestro estudio superan la prevalencia reportada en la población pediátrica chilena en sectores urbano-rurales en los últimos 3 años ${ }^{18,19}$, a los reportes nacionales en niños entre 6 y 12 años entre los años 1996 y $1997^{20}$, y a las estadísticas públicas otorgadas por el Ministerio de Salud de Chile en los años 2007 y 201021,22. Esto refleja el mal estado de salud oral que podría presentar esta población en particular.

El efecto de la DM1, a nivel bucal, se ve reflejado en la alteración en el flujo salival lo que actuaría favoreciendo el proceso de desmineralización dentaria, ya que, es en ésta donde se concentra el flúor necesario para el proceso de remineralización; además, al disminuir su flujo, se disminuye el efecto protector de barrido lo que conlleva en un aumento en la acumulación de placa bacteriana y con ello en el riesgo de caries $^{23,24}$. Dicho aspecto podría verse reflejado y coincidir con los altos índices COPD promedio observado en los pacientes evaluados (mayor al nivel nacional para la edad) $)^{21,22}$.

La presencia de una higiene oral deficiente y la alta prevalencia de caries observada puede deberse a la falta de educación, instrucción y motivación de higiene dada por los pares médicos u odontólogos tratantes, ya que, a pesar de ser una población reducida y controlada, pueden no conocer los métodos correctos de higiene bucal ${ }^{25}$.

La dieta cariogénica juega un rol importante en la producción de la caries. Sin embargo, en nuestro estudio, casi el 100\% de los encuestados obtuvo un potencial cariogénico bajo o moderado, mientras que con un potencial cariogénico alto no se registró ningún caso. $\mathrm{Si}$ bien no se observó una asociación estadística entre el tipo de dieta y la presencia de caries, los resultados no son concluyentes y pueden deberse a la baja proporción de pacientes DM1 con dieta cariogénica tipo moderada. Sin embargo, el riesgo de caries en pacientes diabéti- cos se asocia principalmente a los cambios en la composición y flujo de la saliva (aumento en las concentraciones de glucosa y albumina), un pobre control de la placa bacteriana ${ }^{26,27}$ y un mal control metabólico de la diabetes ${ }^{28}$ tal como se presenta en la población seleccionada en este reporte.

Por ello, se considera de suma importancia establecer programas individuales y grupales de educación temprana y motivación tanto a los propios pacientes, padres o cuidadores como también a médicos tratantes y personal auxiliar, basados principalmente en el control periódico y sistemático por edades y grado de adhesión al tratamiento. Según estudios realizados por Zúñiga et al., ${ }^{29}$ los pacientes diagnosticados con DM1 tienen una mayor tendencia a desarrollar psicopatologías secundarias a su enfermedad que podrían complicar el buen control de su salud general y oral.

En conclusión, se observó un mal estado de salud oral en pacientes con DM1 menores de 15 años. Se sugiere la realización de un control odontológico obligatorio y rutinario asociado al control médico de estos pacientes desde el diagnóstico e inicio del tratamiento. Además, es necesaria la realización de nuevos estudios de índole analítico que asocie factores de riesgo e influencia directa de la fisiopatología DM1 en el estado de salud oral; como también el diseño de estudios clínicos que compruebe la efectividad de intervenciones educativas, tanto a padres, niños y personal auxiliar, en el control de la DM1 y los índices de higiene e historia de caries.

\section{Agradecimientos}

Agradecemos a todo el personal de la unidad de Diabetes del Hospital Regional de Talca y los niños en tratamiento que gentilmente participaron de este estudio.

A los docentes de Clínica Integral del Niño de Escuela de Odontología de Universidad de Talca. A la Dra. Patricia Jiménez del Río por la orientación y apoyo en marco de la investigación para optar al título de Cirujano Dentista de la investigadora principal (XM); y a la Dra. Constanza Fernández, estudiante programa Doctorado en Odontología mención Cariolo- 
gía de la FOP-UNICAMP Brasil, por sus aporte al manuscrito.

\section{Referencias}

1.- Ministerio de Salud. Guía Clínica Diabetes Mellitus Tipo 1. Primera Edición 2005. Santiago. Chile.

2.- Eyzaguirre F, Mericg V: Diabetes Mellitus en el Niño: Una Condición Creciente. Rev Med Clin Condes 2009; 20: 631-4.

3.- Eyzaguirre CF, Peláez DJ, Sepúlveda C, et al: Diabetes Mellitus tipo 1 (DM1) en niños menores de 5 años: Características al debut $v s$ otros grupos etarios en Chile. Rev Chil Pediatr 2006; 77: 375-81.

4.- Saes Busato IM, Bittencourt MS, Machado MA, et al: Association between metabolic control and oral health in adolescents with type 1 diabetes mellitus. Oral Surg Oral Med Oral Pathol Oral Radiol Endod 2010; 109: e51-6.

5.- Asenjo S, Muzzo S, Pérez MV, et al: Consenso en el diagnóstico y tratamiento de la diabetes tipo 1 del niño y del adolescente. Rev Chil Pediatr 2007; 78: 534-41.

6.- Carrasco PE, Angel BB, Codner E, et al: Incidencia de diabetes mellitus tipo 1 en Santiago de Chile: análisis por comunas de la Región Metropolitana en el período 2000-2004. Rev Med Chile 2006; 134: 1258-64.

7.- Moret Y, Muller A, Yuraima P: Manifestaciones bucales de la Diabetes Mellitus Gestacional: Presentación de dos casos y revisión de la literatura. Acta odontol Venez 2002; 40: 160-4.

8.- Finney LS, Finney MO, González-Campoy JM: What the mouth has to say about diabetes. Careful examinations canavert serious complications. Posgrad Med 1997; 102: 117-26.

9.- Miralles L, Silvestre FJ, Grau DM, et al: Estudio clínico sobre la patología bucodentaria en el paciente diabético tipo 1. Medicina Oral 2002; 7: 298-302.

10.- Silvertrs DFJ, Casal CI, Grau GD, et al: Manejo dental de los pacientes discapacitados y médicamente comprometidos. Madrid: Rapport 2000: 1-4.

11.- Psaltopoulou T, Ilias I, Alevizaki M: The Role of Diet and Lifestyle in Primary, Secondary, and Tertiary Diabetes Prevention: A Review of Meta-Analyses. Rev Diabet Stud 2010; 7: 26-35.

12.- Klein H, Palmer CE, Knutson JW: Studies on Dental Caries, I, Dental status and Dental Needs of elementary school. Pub. Health Rep 1938; 53: 751-65.

13.- Gruebbel AO: A measurement of dental caries prevalence and treatment service for deciduous teeth. J Dent Res 1944; 23: 163-8.
14.- Cereceda MA, Faleiros CS, Ormeño QA, et al: Prevalencia de Caries en Alumnos de Educación Básica y su Asociación con el Estado Nutricional. Rev Chil Pediatr 2010; 81: 28-36.

15.- Ministerio de Salud. Guía Clínica Salud Oral integral en niños de 6 años. Primera Edición. Santiago. 2005; 8-10.

16.- Guerrero S, Lipari A: Encuesta de consumo de alimentos cariogénicos en escolares de distintos estratos económicos. Odont Chilena 1992; 40: 61-8.

17.- Servicio de Salud del Maule. Compendio estadístico CENSO 2002. Disponible en: www.ssmaule.cl/Pagina/ Estadisticas/maule.doc. Última revisión 20 abril de 2012.

18.- Cerón A, Castillo V, Aravena P: Caries Prevalence in 10-Year-Old School Children, Frutillar, 2007-2010. Int J Odontostomat 2011; 5: 203-7.

19.- Rivera CA: Pre-school Child Oral Health in a Rural Chilean Community. Int J Odontostomat 2011; 5: 83-6.

20.- Urbina T, Caro JC, Vicent M: Caries dentaria y fluorosis en niños de 6, 8 y 12 años de la II, VI, VIII, IX, X y RM. Chile. División Programas de Salud, Departamento Odontológico Ministerio de Salud, Chile. 1997.

21.- MINSAL, Ceballos M, Acevedo C, et al: Diagnóstico de Salud Bucal de Niños de 2 y 4 años, que asisten a la educación preescolar en la Región Metropolitana, 2007.

22.- MINSAL, Soto L, Tapia R, et al: Diagnóstico Nacional de Salud Bucal del Niño de 6 años, 2007.

23.- Twetman S, Petersson GH, Bratthall D: Caries risk assessment as a predictor of metabolic control in young Type 1 diabetics. Diabet Med 2005; 22: 312-5.

24.- Takahashi N, Nyvad B: Caries Ecology Revisited: Microbial Dynamics and the Caries Process. Caries Res 2008; 42: 409-18.

25.- Moore PA, Orchard T, Guggenheimer J, et al: Diabetes and oral health promotion: a survey of disease prevention behaviors. J Am Dent Assoc 2000; 131: 1333-41.

26.- Siudikiene J, Machiulskiene V, Nyvad B, et al: Dental caries increments and related factors in children with type 1 diabetes mellitus. Caries Res 2008; 42: 354-62.

27.- Siudikiene J, Machiulskiene V, Nyvad B, et al: Dental caries and salivary status in children with type 1 diabetes mellitus, related to the metabolic control of the disease. Eur J Oral Sci 2006; 114: 8-14.

28.- Twetman S, Johansson I, Birkhed D, et al: Caries incidence in young type 1 diabetes mellitus patients in relation to metabolic control and caries-associated risk factors. Caries Res 2002; 36: 31-5.

29.- Zúniga HF, Inzunza CC, Ovalle GC, et al: Diabetes Mellitus Tipo 1 y Psiquiatría Infanto-Juvenil. Rev Chil Pediatr 2009; 80: 467-74. 